\title{
Impact of ureteral access sheath on renal stone treatment: prospective comparative non-randomised outcomes over a 7-year period
}

\author{
Ashleigh Lima ${ }^{1} \cdot$ Thomas Reeves $^{1} \cdot$ Robert Geraghty $^{1} \cdot$ Amelia Pietropaolo $^{1} \cdot$ Lily Whitehurst $^{1} \cdot$ Bhaskar K. Somani $^{1}$ (i)
}

Received: 24 March 2019 / Accepted: 16 July 2019

(c) The Author(s) 2019

\begin{abstract}
Purpose To compare the outcomes (stone free rate and complications) of renal stone treatment with and without the use of ureteral access sheath (UAS). The worldwide use of UAS has risen over the last decade; however, questions still remain on the safety and outcomes with its use. We wanted to look at the role of UAS for treatment of consecutive renal stones over a 7-year period.

Methods The outcomes of flexible ureteroscopy and stone treatment (FURS) for renal stones with and without the use of UAS was prospectively compared from March 2012 to July 2018. Patients were divided into two groups: group-1 where UAS was used for stone treatment and group- 2 where a UAS was not used. Data were collected prospectively on consecutive patients for demographics, stone size, location and number, pre and post-operative stent usage, operative time duration, stone free rate (SFR), length of stay and complications.

Results During the study period, 338 patients underwent FURS for renal stones, of which a UAS was used for 203 (60\%) patients. The mean age of patients was 56 years (range 2-89 years) with a male:female ratio of 204:134. The mean cumulative stone size and the mean number of stones was $16.5 \pm 10.8 \mathrm{~mm}$ and $11.37 \pm 8.08 \mathrm{~mm}(P<0.001)$, and $2.17 \pm 1.99$ and $1.66 \pm 1.50(P=0.009)$ for groups 1 and 2 respectively. The pre and post-operative stent insertion rates were similar in the two groups. The procedural time was longer in group-1 (54.8 $\pm 25.8 \mathrm{~min})$ compared to group-2 (41.3 $\pm 22.2 \mathrm{~min})(P<0.001)$. The SFR for group-1 (88\%) was slightly lower than group-2 (94\%) although this was not statistically significant $(P=0.07)$. There were no intra-operative complications in either of the groups. Post-operative complications were seen in eight patients in group-1 (7 Clavien I/II and 1 Clavien IVa) and two patients in group-2 (Clavien I) $(P=0.19)$.

Conclusion The use of UAS for renal stones is safe with no intra-operative complications noted in our series. Good stone-free rates were obtained for large and multiple renal stones with a small risk of minor complications post-operatively.
\end{abstract}

Keywords Access sheath $\cdot$ Calculi $\cdot$ Laser $\cdot$ Ureteroscopy $\cdot$ RIRS

Bhaskar K. Somani

b.k.somani@soton.ac.uk

Ashleigh Lima

ashleigh_lima@hotmail.com

Thomas Reeves

thomas.reeves@nhs.net

Robert Geraghty

R.Geraghty@soton.ac.uk

Amelia Pietropaolo

ameliapietr@gmail.com

Lily Whitehurst

lilywhitehurst1@hotmail.co.uk

1 Department of Urology, University Hospital Southampton NHS Foundation Trust, Wessex Clinical Research Network and Simulation Lead for Urology, Tremona Road, Southampton SO16 6YD, UK

\section{Introduction}

Ureteric Access Sheath (UAS) are commonly used in endourological procedures especially during flexible ureteroscopy (FURS). They aid in facilitating multiple passage in the kidney, improving irrigation with better fluid outflow thereby improving vision and decreasing the intrarenal pressure [1-9]. There is a perceived advantage of minimising scope damage with it [3]. Conversely, it has been suggested that ureteral wall injuries occur more frequently during ureteroscopy (URS) when using UAS is used [4]. Recorded postoperative complications of FURS with UAS use include ureteric perforation, ischaemia from decreased ureteral blood flow and stricture formation [5]. 
The use of UAS is widely recognised as a technique utilised to keep the intrarenal pressure low when performing FURS for larger and more complex stones, although some authors advocate its use for most renal stones [6-8]. Managing intrarenal pressure seems to prevent acute kidney injury and possibly also reduces the risk of sepsis [10-14]. Operative times greater than $60 \mathrm{~min}$ with irrigation pressure $>20 \mathrm{cmH}_{2} \mathrm{O}$ is linked with renal damage, which is further worsened by peak pressures during forced irrigation, all of which is partly negated with the use of UAS [12]. There is some debate on the size of UAS sheath used with a trend towards using smaller diameter UAS. Although sheath size does influence the irrigation flow, this is much less pronounced when the working channel is occupied $[5,6,15]$.

Ureteric perforation and fluid extravasation can lead to delayed stricture formation especially in the presence of infection [12]. However, a recent study from Anbarasan et al. analysed FURS with UAS in paediatric population and found excellent stone free rate (SFR) with no intra or post-operative complications and no incidence of ureteric stricture on a 26-month follow-up [16]. Although UAS is commonly used in clinical practice, no formal guidelines currently exist [17].

While the size and length of UAS vary depending on the pre-existing ureteral compliance and presence of pre-operative stent, its clinical use usually depends on the surgeon's discretion and patient's anatomy [11]. Advances in technology including miniaturisation of endoscopes will reduce the standard UAS diameter, thereby avoiding the use of oversized UAS. This will prevent complications caused by kinking, buckling and forced insertion [18]. Advances and standardisation of a step-wise technique will further reduce intra operative complications $[19,20]$.

There remains a lack of consensus as to the true impact of UAS on surgical outcomes for renal stones. We hypothesise that the use of an access sheath helps to achieve good SFR for treatment of renal stones without increasing the complication rates. This study aims to report the outcomes of ureteroscopy for treatment of renal stones using an access sheath from a specialist endourology centre in the United Kingdom.

\section{Methods}

Prospective outcomes were recorded for consecutive patients undergoing FURS for renal stones over a period of 7 years between March 2012 and July 2018. The audit was registered in the hospital 'Clinical effectiveness and audit office'. The inclusion criteria were the presence of renal stones of any size or location but patients with ureteric stones or combined renal and ureteric stones were excluded. Outcomes were collected for patients wherein the surgery was performed or supervised by a single surgeon (BS) and analyzed by a third party (RG) not involved in the original procedure. Patients were divided into two groups based on the use of access sheath. Data analysed were patient demographics, stone size, number of stones, length of inpatient stay, operative time, pre- and post-operative stent placement, stone location, stone free rate and post-operative complications. Complications were classified according to the Clavien-Dindo classification [21]. Data were recorded on an excel spreadsheet (Microsoft, USA) and analysed using SPSS version 24. Results are displayed with $P$ values and $95 \%$ confidence intervals (CI), using Chi squared and Fisher's exact test (FET). A $P$ value $<0.05$ was considered statistically significant.

\section{Technique}

The ureteroscopy procedure was performed according to our previously described and validated technique which is used for both endoscopic simulation teaching and clinical use; rigid cystoscopy and placement of a safety guidewire, semirigid ureteroscopy over a working guidewire, placement of an access sheath, and flexible ureterorenoscopy thereafter [16, 19, 20, 22].

Patients were placed in the lithotomy position under general anaesthesia and given appropriate antibiotic cover. The bladder and ureteric orifice were directly visualised by rigid cystoscopy prior to the introduction of a safety guidewire. Following this, a semi-rigid ureteroscopy was performed over a second working wire up to the pelvi-ureteric junction (PUJ) or as far proximally as safely achievable, allowing passive dilatation of the ureteric orifice and ureter. This helped in calibration of the ureter to judge whether an access sheath could be inserted, and an estimate of which size UAS could be safely accommodated. Appropriately sized UAS was then inserted over the working wire and positioned just distal to the PUJ under fluoroscopic guidance. Based on the findings of semi-rigid URS where the ureter was judged to be tight and it was felt that the UAS could not be safely inserted, the FURS was inserted radiologically over a safety wire. The ureter was inspected for damage on withdrawal of the FURS. Stents were placed postoperatively based on the clinical judgement after finishing the procedure.

\section{Equipment}

The access sheath used was a $9.5 / 11 \mathrm{~F}$ or $12 \mathrm{~F} / 14 \mathrm{~F}$ or 14F/16F Cook Flexor UAS sheath (Cook Medical, USA) wherein a size $45 \mathrm{~cm}$ length was used for males, a $35 \mathrm{~cm}$ length was used for females and paediatric patients. Flexible ureteroscopy was done using a 7.5F Flex X2 flexible ureteroscopes [Karl Storz Endoscopy (UK) Ltd.,Slough, UK] with a holmium:YAG laser [20 W or $100 \mathrm{~W}$; Lumenis (UK) Ltd., Elstree, UK] using a 272 micron laser fiber (Lumenis, Inc.). Stone fragment was removed for stone analysis using 
a Cook NGage nitinol stone extractor (Cook Medical, USA). A $6 \mathrm{~F}$ ureteric stent (Cook Medical or Coloplast) was inserted post-operatively in majority of patients and removed a few weeks postoperatively.

\section{Diagnosis and follow-up}

The stone diagnosis was established using a non-contrast CT scan (NCCT) with follow-up based on plain x-ray for radiopaque stones and ultrasound (USS) for radiolucent stones 2-3 months post-ureteroscopy. Stone-free rate was defined using a combination of being endoscopically stone free immediately after FURS and radiologically stone free (defined as fragments $\leq 2 \mathrm{~mm}$ ) on follow-up imaging [22].

Patients were divided into two groups: group-1 where UAS was used for stone treatment and group- 2 where a UAS was not used.

\section{Results}

A total of 338 patients underwent FURS for renal stones, of which UAS was used in $203(60.1 \%)$ patients (Table 1). The access sheath used were 9.5F/11.5F $(n=89,44 \%)$, $12 \mathrm{~F} / 14 \mathrm{~F}(n=110,54 \%)$ and $14 \mathrm{~F} / 16 \mathrm{~F}(n=4,2 \%)$. The mean age of patients was 56 years (range $2-89$ years) with a male:female ratio of 204:134. The mean cumulative stone size and the mean number of stones was $16.5 \pm 10.8 \mathrm{~mm}$ and $11.37 \pm 8.08 \mathrm{~mm}(P<0.001)$, and $2.17 \pm 1.99$ and $1.66 \pm 1.50(P=0.009)$ for groups 1 and 2 , respectively.

The pre-operative stent insertion rates were $27.6 \%$ and $28.3 \%$ with the post-operative stent insertion rates of $82.4 \%$ and $81.1 \%$ in groups 1 and 2, respectively.(Table 1). The procedural time was longer in group-1 $(54.8 \pm 25.8 \mathrm{~min})$ compared to group-2 $(41.3 \pm 22.2 \mathrm{~min})(P<0.001)$. The SFR for group-1 (88\%) was slightly lower than group-2 (94\%) although this was not statistically significant $(P=0.07)$.

There were no intra-operative complications in either of the groups. Eight patients experienced post-operative complications in group-1 with one stent pain (Clavien I), six urinary tract infection or urosepsis (Clavien II) and one urosepsis needing intensive care unit admission (Clavien IVa). Only two patients in group-2 had post-operative complications (Clavien I) with one clot colic and stent related pain each.

\section{Discussion}

To the author's knowledge, this is the largest single-centred prospective study looking at the outcome of FURS for renal stones with and without the use of UAS. While the use of UAS achieved excellent SFR with a low risk of post-operative complications, no intra-operative complications were

Table 1 Patient demographics and outcomes for outcomes of renal stones with and without the use of UAS (ureteral access sheath)

\begin{tabular}{|c|c|c|c|}
\hline FURS for renal stones $(N=338)$ & $\begin{array}{l}\text { Group-1 } \\
\text { UAS used }(n=203)\end{array}$ & $\begin{array}{l}\text { Group - } 2 \\
\text { UAS not used }(n=135)\end{array}$ & $P(95 \% \mathrm{CI})[$ Test $]$ \\
\hline Mean Age & $55.0 \pm 20.3$ & $58.1 \pm 17.8$ & $P=0.15(-1.12$ to 7.33$)$ \\
\hline Gender $(n)$ & $\begin{array}{l}\text { Male: } 117 \\
\text { Female: } 86\end{array}$ & $\begin{array}{l}\text { Male: } 87 \\
\text { Female: } 48\end{array}$ & $P=0.21$ \\
\hline Mean cumulative stone size (mm) & $16.5 \pm 10.8$ & $11.37 \pm 8.08$ & $P<0.001(3.00-7.27)$ \\
\hline Mean number of stones & $2.17 \pm 1.99$ & $1.66 \pm 1.50$ & $P=0.009(0.13-0.89)$ \\
\hline Mean length of stay (days) & $0.82 \pm 5.06$ & $0.22 \pm 0.75$ & $P=0.10(-1.31$ to 0.12$)$ \\
\hline Mean operative time (min) & $54.8 \pm 25.8$ & $41.3 \pm 22.2$ & $P<0.001$ (8.00 to 19.07$)$ \\
\hline Pre-operative stent, $n(\%)$ & $56(27.6 \%)$ & $38(28.3 \%)$ & $P=0.88$ \\
\hline Post-operative stent, $n(\%)$ & $164(82.4 \%)$ & $107(81.1 \%)$ & $P=0.77$ \\
\hline Stone location, $n(\%)$ & $\begin{array}{l}\text { LP: } 81(39.9 \%) \\
\text { MP: } 24(11.8 \%) \\
\text { UP: } 15(7.4 \%) \\
\text { RP: } 28(13.8 \%) \\
\text { Renal NS: } 55(27.1 \%)\end{array}$ & $\begin{array}{l}\text { LP: } 60(44.4 \%) \\
\text { MP: } 14(10.4 \%) \\
\text { UP: } 13(9.6 \%) \\
\text { RP: } 20(14.8 \%) \\
\text { Renal NS: } 28(20.7 \%)\end{array}$ & $P=0.66$ \\
\hline Stone free, $n(\%)$ & $177(88.1 \%)$ & $125(94.0 \%)$ & $\mathrm{P}=0.07$ \\
\hline Complications, $n(\%)$ & $\begin{array}{l}8(4 \%) \\
1 \text { stent pain }(\mathrm{Cl} \mathrm{I}) \\
6 \text { urosepsis }(\mathrm{Cl} \mathrm{II}) \\
1 \text { urosepsis }(\mathrm{Cl} \mathrm{IVa)}\end{array}$ & $\begin{array}{l}2(1.5 \%) \\
1 \text { clot colic }(\mathrm{Cl} \mathrm{I}) \\
1 \text { stent pain }(\mathrm{Cl} \mathrm{I})\end{array}$ & $\mathrm{P}=0.19$ \\
\hline
\end{tabular}

$L P$ lower pole, $M P$ mid pole, $U P$ upper pole, $R P$ renal pelvis, $N S$ not-specified, $C l$ Clavien 
noted with its use. This was partly down to the routine use of semi-rigid URS prior to placing an access sheath allowing passive ureteric dilatation and partly because UAS was not used if the ureter was judged to be tight for a safe insertion of UAS. Similarly, a clinical judgement was made on the size of UAS that could be safely placed. When we compared the two groups, UAS was used in patients with larger or multiple renal stones although there was no difference in the rates of post-operative stent insertion rates between the two groups. This perhaps reflects a clinical bias in the use of UAS for larger more complex renal stones whereas for smaller stones it was not routinely used. The overall SFR with the use of UAS was $88 \%$ and the post-operative complications were $4 \%$, with only one Clavien IVa complication related to urosepsis.

In a previous study, Geraghty et al. looked at the role of UAS for large renal stones with an overall complication rate of $6 \%$, although they found no difference in the SFR or complication rates with and without the use of UAS [23]. The Clinical Research Office of the Endourological Society (CROES) study has been the largest study to date, involving 1494 patients undergoing FURS with UAS [24]. Although no difference in SFR was noted, there was an overall reduction in infection related complications (fever, UTI, sepsis) with the use of UAS. A previous study from Israel showed a failure rate of insertion in $22 \%$ with the use of $14 \mathrm{~F}$ stent and showed a higher success rate in patients with pre-operative ureteric stent, a prior history of ureteroscopy and in the elderly age group [25]. In another retrospective study by Baş et al. the use of UAS was not associated with complications although stone size, multiplicity and congenital renal abnormality were associated with it [26]. Successful placement of UAS was possible in $91 \%$ of patients in a retrospective study of 316 patients by Giusti et al. [27]. After a follow-up of 1-month, the SFR was 79\%, the complication rates were $29 \%$ of which the majority were Clavien I/II complications.

A previous systemic review and meta-analysis by Huang et al. showed no difference in SFR, operative time and intraoperative complications with the use of UAS although the post-operative complications were slightly higher with its use [28]. Our prospective data of consecutive patients with renal stones show similar results in both groups although with a slightly higher operative time in patients with UAS owing to higher stone size, multiplicity and the time taken to insert the sheath itself.

While intra-operative ureteric injuries are mentioned with the use of UAS there does not seem to be any long-term sequelae $[3,29,30]$. Also, subjective difficulty or longer insertion time for sheath placement were associated with high-grade injury, and in such instances a lower threshold was recommended for using a smaller size sheath [29]. To avoid UAS related injury, we recommend a routine semirigid ureteroscopy over an access guidewire in all cases prior to performing a flexible ureteroscopy which could be done with or without an access sheath $[19,20]$. The semi-rigid URS allows passive dilatation of the ureter and allows an estimate on whether it is possible to place a UAS [16]. The latter is only placed if the ureter would allow it, otherwise a FURS is guided over a working guidewire.

Our complication rates with the use of UAS was $4 \%$ but no intra-operative complications were noted compared to CROES global study where $6 \%$ had intra-operative complications including a ureteral perforation rate of $1 \%$ [24]. Although our study includes all patients with renal stones, we did not report on how many patients had a trial of UAS where it was unsuccessful, which was around $1 \%$ in the CROES study. Similarly, being a non-randomised study, patients where UAS was used had larger and multiple renal stones compared to the group where UAS was not used. The imaging modality used for follow-up was mainly plain X-ray or USS rather than a CT scan. The follow-up period was short and perhaps not enough to identify any potential patients with ureteric stricture. Recent research has found that UAS placed under direct or endoscopic vision reduced operative time and radiation exposure to the patient and surgical team, although this work needs further validation [31, 32]. More work also needs to be done on the use of alpha blockers which seems to increase the success with placement of UAS and this needs to be incorporated in future trials [33].

\section{Conclusion}

The use of ureteral access sheath for renal stones is safe with no intra-operative complications noted in our series. Although good stone free rates were obtained in both groups with and without the use of UAS, patients with UAS use had larger or multiple renal stones.

Authors contributions Protocol/project development: AP, BKS. Data collection or management: AP, LW. Data analysis: RG. Manuscript writing/editing: AL, TR, BKS.

Funding No funding was received for this work

\section{Compliance with ethical standards}

Conflict of interest The authors declare that there is no conflict of interest from any of the co-authors

Ethical approval All procedures performed in studies involving human participants were in accordance with the ethical standards of the institutional and/or national research committee and with the 1964 Helsinki Declaration and its later amendments or comparable ethical standards.

Informed consent Informed consent was obtained from all individual participants/parents included in the study. 
Open Access This article is distributed under the terms of the Creative Commons Attribution 4.0 International License (http://creativeco mmons.org/licenses/by/4.0/), which permits unrestricted use, distribution, and reproduction in any medium, provided you give appropriate credit to the original author(s) and the source, provide a link to the Creative Commons license, and indicate if changes were made.

\section{References}

1. Gorin MA, Santos Cortes JA, Kyle CC et al (2015) Initial clinical experience with use of ureteral access sheaths in the diagnosis and treatment of upper tract urothelial carcinoma. Urology 78(3):523-527

2. Miernik A, Schoenthaler M, Wildhelm K et al (2014) Combined semirigid and flexible ureterorenoscopy via a large ureteral access sheath for kidney stones $>2 \mathrm{~cm}$ : a bicentric prospective assessment. World J Urol 32(3):697-702

3. Pietrow PK, Auge BK, Delvecchio FC et al (2002) Techniques to maximize flexible ureteroscope longevity. Urology 60:784-788

4. Traxer O, Thomas A (2013) Prospective evaluation and classification of ureteral wall injuries resulting from insertion of a ureteral access sheath during retrograde intrarenal surgery. J Urol 189:580-584

5. Delvecchio FC, Auge BK, Brizuela RM et al (2003) Assessment of stricture formation with the ureteral access sheath. Urology 61:518-522

6. De Coninck V, Keller EX, Rodriguez-Monsalve M et al (2018) Systematic review of ureteral access sheaths: facts and myths. BJU Int 122(6):959-969

7. Kaplan AG, Lipkin ME, Scales CD Jr et al (2016) Use of ureteral access sheaths in ureteroscopy. Nat Rev Urol 13(3):135-140

8. Breda A, Territo A, Lopez-Martinez JM (2016) Benefits and risks of ureteral access sheaths for retrograde renal access. Curr Opin Urol 26(1):70-75

9. Guzelburc V, Guven S, Boz MY et al (2016) Intraoperative evaluation of ureteral access sheath-related injuries using post-ureteroscopic lesion scale. J Laparoendosc Adv Surg Tech A 26(1):23-26

10. Deng X, Song L, Xie D et al (2016) A novel flexible ureteroscopy with intelligent control of renal pelvic pressure: an initial experience of 93 cases. J Endourol 30(10):1067-1072

11. Wright A, Williams K, Somani B et al (2015) Intrarenal pressure and irrigation flow with commonly used ureteric access sheaths and instruments. Cent Eur J Urol 68(4):434-438

12. Jung H, Osther PJS (2015) Intraluminal pressure profiles during flexible ureterorenoscopy. SpringerPlus 4:373

13. Tokas T, Herrmann TRW, Skolarikos A et al (2019) Pressure matters: intrarenal pressures during normal and pathological conditions, and impact of increased values to renal physiology. World J Urol 37(1):125-131

14. Tokas T, Skolarikos A, Herrmann TRW et al (2019) Pressure matters 2: intrarenal pressure ranges during upper-tract endourological procedures. World J Urol 37(1):133-142

15. Ng YH, Somani BK, Dennison A et al (2010) Irrigant flow and intrarenal pressure during flexible ureteroscopy: the effect of different access sheaths, working channel instruments, and hydrostatic pressure. J Endourol 24(12):1915-1920

16. Anbarasan R, Griffin SJ, Somani BK (2019) Outcomes and longterm follow-up with the use of ureteral access sheath for pediatric ureteroscopy and stone treatment: results from a tertiary endourology center. J Endourol 33(2):79-83

17. Türk C, Petřík A, Sarica K et al (2016) EAU Guidelines on diagnosis and conservative management of urolithiasis. Eur Urol 69(3):468-474
18. Al-Qahtani SM, Letendre J, Thomas A et al (2014) Which ureteral access sheath is compatible with your flexible ureteroscope? J Endourol 28(3):286-290

19. Veneziano D, Ahmed K, Cleyenbreugel V et al (2011) Development methodology of the novel endoscopic stone treatment step 1 training/assessment curriculum: an international collaborative work by European Association of Urology Sections. J Endourol 31:934-941

20. Veneziano D, Ploumidis A, Proietti S et al (2018) Evolution and Uptake of the Endoscopic Stone Treatment Step 1 (EST-s1) Protocol: establishment, validation and assessment in a collaboration by the European School of Urology and the Uro-Technology and Urolithiasis Sections. Eur Urol 74(3):401-402

21. Mitropoulos D, Artibani W, Biyani CS et al (2018) Validation of the Clavien-Dindo grading system in urology by the european association of urology guidelines ad hoc panel. Eur Urol Focus 4(4):608-613

22. Ghosh A, Oliver R, Way C et al (2017) Results of day-case ureterorenoscopy (DC-URS) for stone disease: prospective outcomes over 4.5 years. World J Urol 35(11):1757-1764

23. Geraghty RM, Ishii H, Somani BK (2016) Outcomes of flexible ureteroscopy and laser fragmentation for treatment of large renal stones with and without the use of ureteral access sheaths: results from a university hospital with a review of literature. Scand J Urol 50(3):216-219

24. Traxer O, Wendt-Nordahl G, Sodha H et al (2015) Differences in renal stone treatment and outcomes for patients treated either with or without the support of a ureteral access sheath: the Clinical Research Office of the Endourological Society Ureteroscopy Global Study. World J Urol 33(12):2137-2144

25. Mogilevkin Y, Sofer M, Margel D, Greenstein A, Lifshitz D et al (2014) Predicting an effective ureteral access sheath insertion: a bicenter prospective study. J Endourol. 28(12):1414-1417

26. Baş O, Tuygun C, Dede O et al (2017) Factors affecting complication rates of retrograde flexible ureterorenoscopy: analysis of 1571 procedures-a single-centre experience. World J Urol 35:819-826

27. Giusti G, Proietti S, Villa L et al (2016) Current standard technique for modern flexible ureteroscopy: tips and tricks. Eur Urol 70(1):188-194

28. Huang J, Zhao Z, AlSmadi JK et al (2018) Use of the ureteral access sheath during ureteroscopy: a systematic review and metaanalysis. PLoS One 13(2):e0193600

29. Traxer O, Thomas A (2013) Prospective evaluation and classification of ureteral wall injuries resulting from insertion of a ureteral access sheath during retrograde intrarenal surgery. J Urol 189(2):580-584

30. Loftus CJ, Ganesan V, Traxer O et al (2019) Ureteral wall injury with ureteral access sheaths: a randomised prospective trial. J Endourol. https://doi.org/10.1089/end.2018.0603 (Epub ahead of print)

31. Aghamir SMK, Salavati A (2018) Endovisually guided zero radiation ureteral access sheath placement during ureterorenoscopy. Minim Invasive Ther Allied Technol 27(3):143-147

32. Karabulut I, Keskin E, Bedir F et al (2016) Rigid ureteroscope aided insertion of ureteral access sheath in retrograde intrarenal surgery. Urology 91:222-225

33. Koo KC, Yoon JH, Park NC et al (2018) The impact of preoperative $\alpha$-adrenergic antagonists on ureteral access sheath insertion force and the upper limit of force required to avoid ureteral mucosal injury: a randomized controlled study. J Urol 199(6):1622-1630. https://doi.org/10.1016/j.juro.2017.09.173 Epub 2018 Feb 2

Publisher's Note Springer Nature remains neutral with regard to jurisdictional claims in published maps and institutional affiliations. 